\title{
La démarche qualité en Australie
}

Un prisme des transformations universitaires

Quality control in Australia. A prism of academic transformations

El enfoque de calidad de Australia. Un prisma de las transformaciones

universitarias

\section{Martin Carroll et Josephine Palermo}

Traducteur : Cécile Boyer

\section{OpenEdition}

\section{Journals}

Édition électronique

URL : http://journals.openedition.org/ries/265

DOI : $10.4000 /$ ries.265

ISSN : 2261-4265

\section{Éditeur}

Centre international d'études pédagogiques

\section{Édition imprimée}

Date de publication : 1 septembre 2007

Pagination : 111-125

ISBN : 978-2-85420-570-1

ISSN : $1254-4590$

Référence électronique

Martin Carroll et Josephine Palermo, "La démarche qualité en Australie », Revue internationale d'éducation de Sèvres [En ligne], 45 | septembre 2007, mis en ligne le 23 juin 2011, consulté le 01 mai 2019. URL : http://journals.openedition.org/ries/265 ; DOI : 10.4000/ries.265 


\title{
La démarche qualité en Australie
}

\section{Un prisme des transformations universitaires*}

\author{
Martin Carroll \\ Josephine Palermo
}

En Australie, les universités sont soumises à des audits de qualité externes par l'Agence pour la qualité des universités australiennes (Australian Universities Quality Agency : $A U Q A^{1}$ ) qui contrôle leur politique et leurs pratiques en matière de qualité. Entre 2002 et 2007, l'AUQA a mené ses premiers audits selon la méthode dite des «moyens appropriés ${ }^{2}$ dans la presque totalité des universités du pays ${ }^{3}$. La somme des trente-huit rapports d'audits qui en résulte constitue une évaluation exhaustive ${ }^{4}$, et validée par un organisme indépendant, de l'état des activités universitaires. Bien qu'il n'y ait pas de recette préconisée ou de critère prédéfini pour ces audits, tous comportaient un volet sur la recherche et la formation à la recherche (c'est-à-dire des études de second cycle ${ }^{5}$ comprenant une grande part de recherche).

Cet article propose une analyse des conclusions des audits. Une brève analyse critique du cadre réglementaire de la recherche en Australie sera d'abord développée. Si les audits de l'AUQA se sont déroulés pendant une période de relative stabilité en ce qui concerne les systèmes de financement, des changements de politiques importants sont survenus dans le même temps, et un Cadre de qualité de la recherche a notamment été élaboré.

\section{DES ChANGEMENTS DE STRUCTURES DE FINANCEMENT}

Comme dans d'autres pays (Jongbloed \& Vossensteyn, 2001), l'une des réformes majeures de l'enseignement supérieur entreprise par le gouvernement australien au début des années quatre-vingts fut de changer la structure et le

\footnotetext{
*Article traduit par Cécile Boyer.

1. Voir http://www.auqa.edu.au/ (dernier accès au site le 3 juillet 2007).

2. Cela signifie que plutôt que de mener une évaluation en se référant à des critères standards externes imposés, les audits se réfèrent principalement aux déclarations d'intention de l'université évaluée (mission, vision, valeurs, visées, objectifs, cibles, etc.). En conséquence, les conclusions de l'audit s'attachent spécifiquement aux pratiques effectives de l'université et à ce qu'elle considère comme important. Elles ne se contentent pas de refléter un contexte de recherche de manière prédéterminée selon des normes prédéfinies.

3. L'AUQA a aussi mené des audits dans diverses autres entités, par exemple d'autres institutions accréditées, des agences d'accréditation d'État et territoriales et un petit nombre d'institutions non-accréditées.

4. À l'exception de l'Australian National University, dont les résultats d'audit n'étaient pas disponibles lors de la rédaction de cet article.

5. Les auteurs entendent ici par second cycle, comme dans tout l'article, la formation pré-doctorale et doctorale.
} 
processus de financement des universités. Le financement basé uniquement sur les résultats passés des établissements fut remplacé par le Modèle de financement relatif (Relative Funding Model : RFM) à la fin des années quatre-vingts, dans le but de générer de la compétitivité entre les établissements et d'améliorer leur productivité et leur efficacité. Cependant, au cours des années suivantes, le nombre d'étudiants poursuivant des études supérieures s'accrut rapidement, et de manière disproportionnée par rapport à l'accroissement des dépenses publiques pour financer le fonctionnement des universités. Les universités furent donc contraintes de diversifier leurs ressources en développant des activités entrepreneuriales pour pouvoir faire face à la détérioration de leurs infrastructures et à un ratio personnel/étudiants de plus en plus déséquilibré. Avec le passage soudain à un enseignement supérieur accueillant un très large public, et non plus seulement une élite, le financement intégral du fonctionnement des universités par le gouvernement devint extrêmement difficile. Afin d'inciter les universités à diversifier leurs modes de financement, et de les rendre, ce faisant, moins dépendantes du financement public, le gouvernement a élaboré un système dans lequel les fonds publics sont alloués aux universités d'après une forme d'évaluation de leurs performances (Liefner 2003; Geuna \& Martin, 2003).

En Australie, contrairement à l'enseignement et à l'apprentissage, la recherche s'est toujours efforcée d'obtenir des résultats pour justifier les ressources qui lui sont allouées. Le gouvernement fédéral propose divers types de subventions aux universités ${ }^{6}$, dont les principaux, pendant la période des audits, furent les Research Infrastructure Block Grants (RIBG), le Research Training Scheme (RTS), l'Institutional Grants Scheme (IGS). De plus, il existait deux autres grands systèmes de subventions (et divers autres) allouées à la recherche compétitive. Le premier fonds (et le plus important) est administré par le Conseil australien de la recherche ${ }^{7}$, et le deuxième par le Conseil national de la santé et de la recherche médicale ${ }^{8}$.

Alors que ces mécanismes de financement se fondent en partie sur l'évaluation de la performance (en particulier le RTS qui utilise des mesures quantitatives comme gage de qualité), il n'existe pas à ce jour de grille d'évaluation standard pour mesurer (et comparer) de manière systématique la qualité et l'impact des recherches menées dans les universités australiennes. En particulier, il n'existe aucune base de données pour évaluer dans quelle mesure la recherche profite à la communauté australienne dans son ensemble.

Pour répondre à cette lacune, le Cadre de qualité de la recherche (Research Quality Framework: $R Q F$ ) a récemment été mis en place dans

6. Voir http://www.dest.gov.au/sectors/research_sector:programmes_funding/programmes_fundings_menu.htm (denier accès au site le 3 juillet 2007).

7. Voir http://www.arc.gov.au/ (dernière mise à jour le 3 juillet 2007).

8. Voir http://www.nhmrc.gov.au/ (dernière mise à jour le 3 juillet 2007). 
l'enseignement supérieur. Son but est de s'assurer que la recherche est de la plus grande qualité et qu'elle bénéficie à la communauté dans son ensemble, et pas seulement à l'enseignement supérieur (RQFDAG, 2006). Le RQF doit commencer ces premières évaluations en 2008. Il se focalisera sur la qualité de la recherche originale, évaluant son mérite académique intrinsèque reconnu par ses pairs, ainsi que son impact sur la communauté en termes de débouchés sociaux, économiques, environnementaux ou culturels.

Le $R Q F$ ressemble à l'Exercice d'évaluation de la recherche (Research Assessment Exercise : RAE) utilisé en Grande-Bretagne car il a recours au bilan effectué par la communauté des chercheurs dans sa méthode d'évaluation. Les établissements soumettent leurs dossiers à un panel de treize experts (panel d'évaluation) qui les évalue et les note. Mais, contrairement au $R A E$, avec le $R Q F$, la qualité et l'impact sont évalués séparément, selon différents types de critères.

Les panels d'évaluation évalueront les résultats des équipes de chercheurs sélectionnées par l'établissement. Le dossier présenté inclura les résultats des quatre équipes " éligibles» (terme à l'origine de vifs débats) pour recevoir une subvention, ainsi que la liste complète des travaux menés par chaque équipe de recherche. Chaque équipe de recherche devra fournir une sorte d'état des lieux du contexte de la recherche (Context Statement) mentionnant le type, la composition et le domaine de recherche de l'équipe; des informations supplémentaires d'ordre factuel démontrant le potentiel de l'équipe (comme le nombre total de résultats obtenus par catégories de recherches, la preuve d'une collaboration active, les diverses publications, le nombre de chercheurs purs (non enseignants), de jeunes chercheurs, de thésards, de personnes ayant soutenu leur thèse, etc., les emplois auxquels se destinent les étudiants chercheurs, la réputation des membres à travers leurs distinctions; et enfin les subventions obtenues par l'équipe du fait de sa compétitivité.

Un financement basé sur la performance tend à apporter des changements positifs, mais est aussi source d'effets indésirables imprévus. Les formes d'allocations de ressources influencent le comportement des universitaires et des administrateurs de l'enseignement supérieur, surtout en ce qui concerne leur niveau d'activité, les types d'activités dans lesquelles ils s'engagent, et leur façon de gérer les risques. Selon Jongbloed et Vossensteyn (2001), les indicateurs de performance peuvent conduire à des comportements d'évitement de la prise de risque chez les administrateurs des institutions et chez les universitaires parce que les résultats faciles à obtenir seront forcément recherchés en premier.

Cela apparaît nettement lorsque l'on regarde le comportement adopté en Australie avec un mécanisme d'allocation de recherche fondé sur la performance, comme le Research Management Framework qui met l'accent sur le volume de la recherche en tant que critère de performance pour allouer $10 \%$ des subventions de fonctionnement. Du fait de ce mécanisme, les universitaires 
d'Australie ont multiplié par dix leur production de résultats en matière de recherche, mais cela n'a pas entraîné une augmentation proportionnelle de l'impact de la recherche. En d'autres termes, les universitaires australiens ont produit plus de recherche, mais cela s'est traduit par plus de publications dans des revues médiocres, celles-ci étant plus faciles à intéresser que des revues spécialisées de premier ordre.

On ne sait pas encore dans quelle mesure le $R Q F$ infléchira cette tendance. Alors que le gouvernement affiche sa volonté d'encourager la collaboration entre les chercheurs en soutenant une véritable culture de la recherche, le système qui consiste à allouer plus de fonds aux établissements obtenant les meilleurs résultats créera sans nul doute un esprit de compétition, plus que de coopération, entre les chercheurs. Des cas de débauchages se sont déjà produits : les universités bien dotées financièrement cherchent à recruter la crème des chercheurs travaillant dans des établissements moins prestigieux.

\section{ANALYSE DES CONCLUSIONS DE L'AUDIT}

\section{La planification de la recherche}

Les universités font en général une bonne planification centralisée de la recherche. Celle-ci leur est en partie dictée par les exigences du gouvernement fédéral en matière de gestion des projets de recherche et de formation à la recherche (Research and Research Training Management Plans ${ }^{9}$ ). Cependant, certaines feuilles de routes sont excessivement complexes, et d'autres s'articulent mal avec les projets au niveau des facultés. Une analyse thématique des objectifs de haut niveau des universités laisse apparaître des points communs :

- le maintien et l'amélioration du contrôle de qualité;

- un développement croissant des débouchés et des revenus de la recherche;

- l'augmentation du nombre d'étudiants chercheurs et la réduction du temps nécessaire pour terminer leurs études;

- la création de partenariats entre la recherche et certains groupes spécifiques (les professions libérales, l'industrie, etc.);

- le recrutement et/ou la formation d'excellents chercheurs.

Comme on pouvait sans doute le prévoir, l'une des grandes différences entre les universités porte sur le niveau de reconnaissance recherché : alors que

9. Une partie du Cadre d'évaluation des universités du gouvernement fédéral. Non exigé en 2007, mais voir http://www.dest.gov.au/sectors/higher_education/ (dernier accès au site le 3 juillet 2007). 
les universités du Go8 ${ }^{10}$ sont en quête d'une stature internationale, les autres universités recherchent une reconnaissance nationale, voire régionale.

Une autre différence réside dans la façon dont les universités décrivent le contexte universitaire dans lequel elles s'inscrivent. Beaucoup d'universités n'appartenant pas au Go8 ont accédé au statut d'université entre 1989 et 1992, en conséquence de la fusion d'établissements qui n'avaient pas été créés pour faire à la fois de la recherche et de l'enseignement. Dans ces conditions, de nombreuses universités considèrent que le début de leur mission de recherche remonte à l'époque des fusions. C'est pourquoi l'analyse qu'elles font de leur mission de recherche débute souvent par «partant de relativement bas...». Peutêtre, alors, n'est-il pas surprenant de constater que les rapports d'audit de douze universités (39\%) n'appartenant pas au Go8 relatent le besoin d'améliorer les conditions/la culture de la recherche pour la faculté et/ou les étudiants chercheurs, en organisant, par exemple, plus de séminaires et en promouvant le développement des activités de recherche interdisciplinaires. Ce problème concerne particulièrement les campus annexes - ainsi que les universités du Go8.

Une préoccupation commune à toutes les universités, anciennes et nouvelles, est leur besoin de centrer leurs activités de recherche sur un nombre de domaines restreints. Cela est en partie motivé par les politiques de financement du gouvernement fédéral. Presque toutes les universités ont défini un petit nombre de domaines où leurs recherches se concentrent (domaines définis dans leurs grandes lignes en règle générale), et octroient en priorité des crédits aux recherches dans ces domaines. Plusieurs universités ont déclaré que plus de $80 \%$ de leurs activités de recherche appartenaient à ces pôles privilégiés où se concentrent leurs recherches. Certaines universités ont décidé de choisir ces domaines en fonction de leurs points forts actuels, c'est-à-dire les domaines ayant amené le plus de résultats et de revenus. Pour d'autres, c'est un mélange de points forts et de domaines prometteurs afin de s'assurer que la physionomie de la recherche reste dynamique.

De façon intéressante, l'alignement des pôles de recherche et des programmes d'enseignement paraît largement fortuit, parce que les pôles ne sont pas déterminés en fonction des programmes universitaires (considérés comme des facteurs non déterminants).

La mise en place de pôles d'excellence de concentration de recherche a permis d'instaurer une organisation plus coordonnée. Néanmoins, cela a aussi

10. Les universités les plus anciennes, qui mènent des recherches de manière intensive, sont communément appelées le «Groupe des Huit» (Go8) : l'Université d'Adélaïde, l'Université de Sydney, l'Université de Western Australia, l'Université de Tasmanie, l'Université Monash, l'Université de New South Wales, l'Université de Melbourne. Le Go8 (entre autres critères) détient $70 \%$ des subventions nationales pour la recherche compétitive, mène $60 \%$ de la recherche universitaire, détient deux tiers des brevets, et est responsable de la création de pratiquement $80 \%$ des sociétés issues de la recherche des universités australiennes. Il s'agit aussi d'établissements beaucoup plus anciens, ce qui signifie qu'ils ont disposé de plus de temps pour ancrer le contrôle de la qualité dans leurs pratiques et pour générer les conditions propices à une recherche de très haute qualité. 
créé des tensions entre d'une part les domaines de recherche spécialisés, et d'autre part, le fait de devoir maintenir des activités de recherche dynamiques dans tous les domaines d'enseignement.

Ce problème a été identifié dans onze rapports d'audit, dont dix portant sur des universités n'appartenant pas au Go8 (il faut peut-être y voir le signe que, n'ayant pas les reins aussi solides, financièrement, que les universités du Go8, elles se sentent menacées d'être obligées de réduire/perdre la diversité des disciplines qu'elles enseignent). Ce point revêt une importance cruciale au regard des méthodes d'allocation des fonds : un département universitaire ne faisant pas partie des pôles de concentration de recherche rencontre plus de difficultés pour obtenir des fonds. Dans son rapport d'audit (2004), l'Université de New England affirme que «cette stratégie est difficile à défendre d'un point de vue culturel, puisqu'elle semble marginaliser un certain nombre d'équipes actives de recherche».

Nous pensons que les méthodes élaborées pour l'évaluation par le Cadre de qualité de la recherche vont encourager un encore plus grand effort de concentration de la recherche, et ce au détriment d'un certain nombre d'équipes actives de recherche et d'une offre de formation et d'apprentissage ouverte au plus grand nombre.

Afin de savoir si elles réussissent à atteindre leurs objectifs stratégiques, les universités doivent pouvoir se référer à un ensemble de critères d'évaluation de la performance, qui, s'ils doivent être les plus simples possibles, ne doivent pas non plus être trop simplifiés. Cela s'avère grandement problématique. Certaines mesures quantitatives, telles que la classification des revenus de la recherche, sont bien établies. D’autres, comme la classification des résultats de la recherche, sont en place depuis quelques temps mais doivent remporter de nouveaux défis : par exemple, comment classer les résultats de la recherche créative indigène? Il faut également établir de nouveaux ensemble de données pour permettre le contrôle des nouveaux objectifs stratégiques (comme l'alignement de la recherche sur les besoins de l'industrie, des professions libérales, des communautés), ou pour pouvoir mesurer l'impact de la recherche, et évaluer l'efficacité des partenariats. Dix-neuf universités (50\%) ont constaté des lacunes dans les bases de données existantes (et compte tenu du champ d'étude très circonscrit de cette analyse, elles sont sans doute plus nombreuses en réalité).

Toutes les universités sont aussi attentives à la façon dont l'interprétation de telles données pourra être facilitée par un système d'évaluation approprié, et, dans une certaine mesure, s'engagent dans un processus d'évaluation de la gestion de la recherche. Celles qui sont membres de consortiums s'en sortent le mieux à cet égard (et plus particulièrement les universités du Go8 et celles du réseau technologique australien des universités $\left.{ }^{11}\right)$.

11. Australian Technology Network of universities (ATN) : voir http://www.atn.edu.au/ (consulté le 2 juillet 2007). 


\section{L'animation de la recherche}

Quasiment toutes les universités sont dotées d'un comité de la recherche responsable des politiques et stratégies de la recherche. Ces comités sont généralement composés de membres du conseil d'administration de l'université, mais ils n'ont parfois qu'un pouvoir de conseil auprès du vice chancelier. Les membres de ces comités émanent des facultés. Selon les universités, il existe toute une gamme d'autres comités liés à la recherche (qui s'occupent de l'éthique, de la valorisation, etc.).

Dans presque toutes les universités, on trouve au moins un administrateur confirmé responsable de l'organisation de la recherche. Dans les grandes universités, c'est souvent un adjoint du vice chancelier; dans les universités plus récentes et plus petites, c'est souvent le vice chancelier lui-même. Parfois, ils gèrent d'autres portefeuilles comme l'innovation, la valorisation, l'entreprenariat, l'internationalisation, et plus rarement l'enseignement. Ces autres portefeuilles témoignent bien de l'orientation de plus en plus commerciale adoptée par la gestion des activités de recherche.

Dans les facultés et collèges universitaires, un doyen associé est souvent désigné comme responsable de la recherche. D'après les conclusions de l'audit, les politiques de recherche sont menées de manière très efficace lorsqu'une structure commune d'organisation de la recherche coordonne toute l'université, et lorsque des doyens associés (pour la recherche), ou leur équivalent, sont membres du comité de recherche.

La structure de base pour soutenir la recherche est le centre de recherches. La plupart des universités ont un réseau hiérarchisé de centres. S'il n'existe pas de norme (autres que les structures spécifiquement financées par le gouvernement fédéral comme les centres de recherche en coopération ${ }^{12}$ ), on rencontre fréquemment le système de hiérarchisation suivant, du plus haut au plus bas : les instituts, les centres et les équipes de recherche.

Les instituts sont sensés générer des revenus externes conséquents liés à la recherche, et l'université est sensée y investir beaucoup (par le financement, l'attribution d'un directeur à plein temps, l'attribution de bourses de recherche, etc.). Un centre sera probablement plus petit, avec des visées et un soutien moindres. On incite les instituts et les centres à former des partenariats et des liens avec d'autres entités de recherche, dans le secteur industriel ou libéral. La plupart du temps, les instituts et les centres se trouvent dans l'enceinte de la faculté et rendent compte de leurs activités au doyen, mais lorsqu'ils sont de nature interdisciplinaire, ils peuvent se trouver en dehors de la faculté et dépendre de l'adjoint au vice chancelier ou du vice chancelier lui-même. Il y a des exceptions, comme à l'Université de Wollongong, où tous les centres résident dans l'enceinte d'une faculté, mais dépendent du vice chancelier en charge de la recherche.

12. Voir http://www.crc.gov.au/Information/ (dernier accès au site le 3 juillet 2007). 
Une université peut par exemple provisionner un financement sur deux ans, le temps de déterminer si l'équipe de recherche a un potentiel réalisable.

Nul doute que la masse critique de capacité et de soutien engendrée par les instituts et les centres permet de mener de meilleures recherches : les rapports d'audit confirment ce point pour toutes les universités d'Australie. Dans certains cas, cependant, les universités n’ont pas évalué leurs instituts ou centres, ou l'ont fait en utilisant des méthodes qui requièrent une attention particulière, ce qui a empêché les rapports de pouvoir affirmer la qualité et la viabilité des programmes de recherche. Beaucoup d'universités sont en train de réviser leurs critères d'évaluation des instituts et centres (en partie pour anticiper l'évolution du Cadre de qualité de la recherche).

Quelques universités (du Go8 ou non) font appel à des comités de conseillers comprenant des membres ne faisant pas partie de leurs instituts et centres. Parfois, ces comités ont été décriés, dépeints comme des artifices plutôt que comme de réels facteurs d'efficacité du système de contrôle de la qualité de la recherche. Dans d'autres cas, il a été souligné qu'ils « concourent à l'orientation générale de la recherche et aident le monde de la recherche à tisser des liens avec les communautés extérieures concernées ${ }^{13}$.

\section{L'efficacité de la recherche}

La productivité de la recherche dans les universités australiennes est impressionnante et en plein essor. On constate une tendance à son augmentation dans presque toutes les universités (même si les revenus de la recherche varient puisque chaque établissement, dans un même secteur, joue des coudes pour obtenir chaque année une part du marché). Il est intéressant de noter que, bien que les universités aient publiquement et invariablement condamné l'utilisation d'un classement en matière de performance de la recherche (et à raison, selon nous), la plupart ont fait référence à leurs bons classements lorsqu'elles se sont soumises à l'audit.

L'un des indicateurs pour mener de telles comparaisons est la proportion de personnel pouvant être qualifié «d'actif dans la recherche». D'après les rapports d'audit, la définition exacte de cette expression s'avère extrêmement problématique dans la mesure où de subtiles distinctions peuvent au final créer de grandes différences dans les résultats des calculs. Une stratégie efficace, et recommandée à plusieurs reprises dans les rapports d'audit, consiste à appliquer des indices d'activité de la recherche : cela rend les définitions claires et lisibles et permet à la faculté est de planifier au mieux son travail.

De nombreuses universités (et notamment celles du Go8) concentrent leurs efforts sur le soutien aux facultés et à leurs demandes d'attributions de subventions de compétitivité externes, à travers des bureaux de recherche

13. Rapport d'audit de l'AUQA pour l'Université New England, 2004, p. 31. 
centralisés. Cette assistance inclut généralement une aide pour constituer le dossier de demande de subvention (souvent avec le concours d'un chercheur plus expérimenté), mais, la plupart du temps, cela inclut aussi un processus d'évaluation préalable. Les universités plus récentes, au contraire, concentrent leur soutien aux facultés sur l'aide à la reconnaissance de leur programme doctoral et postdoctoral.

La charge de travail dans les facultés est un sujet de préoccupation. Beaucoup de personnels ont déclaré ne pas avoir suffisamment de temps pour mener une recherche, répondre aux attentes en matière de supervision de la Higheer Degrees Research $(H D R)^{14}$, et pour se consacrer à l'enseignement, pourtant considéré comme l'activité la plus importante. Sept universités (mais aucune du Go8) ont fait part de ce problème.

\section{La valorisation de la recherche}

La plupart des universités reconnaissent l'importance vitale de la valorisation de la recherche, y compris celles qui n'ont pas encore une activité de recherche très développée. Un quart environ des rapports d'audit mentionnent que l'université évaluée essaie d'améliorer ses politiques en matière de respect de la propriété intellectuelle, domaine qui semble poser problème, vraisemblablement du fait des intérêts financiers en jeu pour toutes les parties concernées.

Près de la moitié des rapports d'audit mentionnent que l'université évaluée a mis en place une entité chargée de gérer et faciliter la valorisation de la recherche. Dans la moitié des cas (neuf), cette entité a le statut de société commerciale. Cela indique clairement le souci croissant pour la recherche d'attirer des fonds privés pour générer des revenus, en partie dans le but de réduire leur dépendance vis-à-vis des caisses de l'État. La plupart du temps, cette stratégie a porté ses fruits et elle a parfois été félicitée par l'AUQA. Plus encore, l'AUQA a notifié aux deux universités jusqu'ici réfractaires à ces notions de reconsidérer leur position.

La création de parcs de recherche constitue l'une des stratégies les plus importantes pour encourager la valorisation de la recherche par le biais de relations avec l'industrie. L'AUQA atteste de l'instauration de cette stratégie déterminante dans quatre universités (Adélaïde, Macquarie, La Trobe et Wollongong). Même si l'AUQA souligne l'importance de l'investissement et les bénéfices potentiels, elle rappelle qu'il faut prêter une très grande attention à la façon dont les activités du parc de recherche, de l'enseignement général et des Higheer Degrees by Research (HDR) de l'université doivent s'articuler, pour qu'un bénéfice maximum soit tiré d'une telle structure.

14. Équivalent de quoi dans le système français ? ??? Voir § suivant. 


\section{LA FORMATION À LA RECHERCHE Organisation}

Il y a dans toutes les universités australiennes des étudiants poursuivant des études de second cycle comprenant une grande part de recherche $(H D R)$ et ce jusqu'au niveau doctoral ${ }^{15}$.

Presque toutes les universités ont un comité du second cycle (Higher Degree Committee) dont l'appellation peut varier (Research Studies Committee, Research Training Committee, Graduate Studies Committee, etc.), chargé des politiques et stratégies en matière de formation à la recherche. Il s'agit généralement d'un sous-groupe du comité de la recherche émanant des facultés. Toute une gamme d'autres comités eux aussi liés à la formation à la recherche existe (pour les bourses, etc.). Cela varie beaucoup d'une université à l'autre.

L'administrateur chargé de l'organisation de la recherche est presque toujours celui qui est chargé de l'organisation de la formation à la recherche. $\mathrm{Au}$ sein des facultés et des collèges universitaires, la responsabilité de l'organisation repose souvent sur un doyen associé (pour le premier cycle) ou sur le doyen associé (pour la recherche). Au niveau supérieur de l'école/du département, la tendance est de plus en plus à la désignation de coordinateurs du second cycle qui ont pour responsabilité (outre leur responsabilité de supervision) d'assurer l'interface directe avec les étudiants en $H D R$.

On a déjà relevé le besoin général d'intensifier le climat de recherche pour les étudiants en $H D R$. Pour atteindre cet objectif, une tendance nette a été de remplacer les traditionnels bureaux des études de second cycle par les nouvelles Graduate Schools. Chaque Graduate School est dirigée par un doyen, qui dote cette structure d'un statut académique et d'une reconnaissance, et qui assure, par sa présence, qu'un représentant des questions relevant des $H D R$ assistera à la réunion des doyens. Douze universités (32\%) ont évolué dans ce sens.

Un rapport d'audit confirme que «le rôle de l'Université de Technologie de Sydney est de "promouvoir l'excellence et l'innovation dans l'enseignement de la recherche" ainsi que de gérer les processus de candidature des étudiantschercheurs ${ }^{16}$.

Malgré les craintes exprimées initialement qu'une telle structure ne finisse par empiéter sur le rôle des écoles/départements, cette évolution semble produire des résultats positifs et se poursuivra vraisemblablement. L'AUQA a formellement, et à plusieurs reprises, recommandé pour leur efficacité les Graduate Schools administrées par des doyens.

15. Il existe aussi des étudiants de second cycle qui suivent simplement des cours, mais ils ne font pas partie de notre étude.

16. Rapport d'audit de l'AUQA pour l'Université de Technologie de Sydney, 2006, p. 45. 
La fonction de doyen, en particulier, s'avère utile à la fois pour harmoniser les politiques et les pratiques, et en tant que référent spécifique des associations d'étudiants du second cycle.

\section{Contenus et finalités}

Toutes les universités disposent de mesures (souvent appelées «codes de pratique») qui guident la recherche de second cycle et l'encadrement du second cycle. Ces mesures varient considérablement en substance et en efficacité, mais sont pour la plupart très utiles, et s'avèrent parfois être des ressources exceptionnelles. Trois facteurs essentiels s'en dégagent et demandent qu'on y attache une grande attention : la progression des étudiants, leur satisfaction, et la qualité des directeurs de thèse.

Presque toutes les universités ont un dispositif pour contrôler régulièrement le niveau d'avancement des étudiants chercheurs et de leurs directeurs de recherche. La fréquence à laquelle les bilans doivent être effectués a donné lieu à de nombreux débats. Treize universités fonctionnent avec un système de compte rendu annuel, sept avec un compte rendu biannuel (et quelques autres avec des systèmes différents). Quoi qu'il en soit, les rapports d'audit ne relèvent pas de différence flagrante en ce qui concerne l'efficacité de ces deux modes de fonctionnement (d'ailleurs, la seule existence d'un tel système ne suffit pas à garantir son efficacité). Ce qui est crucial, c'est plutôt, semble-t-il, l'exploitation qui est faite des comptes rendus pour contrôler les politiques du $H D R$ et, à cet égard, un petit nombre d'universités ne tire pas le meilleur profit de ces informations.

Un problème récurrent souligné par les rapports d'audit est l'absence de procédure de doléance simple et claire par laquelle les étudiants pourraient faire part de leurs griefs vis-à-vis de leur directeur. Lorsque ces procédures de doléance ne sont pas adéquates, le compte rendu de progression de l'étudiant s'y substitue, étant perçu par les personnels et par les étudiants comme un système par défaut, bien qu'il ne soit pas destiné à cet usage. En fait, le caractère inapproprié des moyens instaurés pour contrôler la qualité de la direction des $H D R$ apparaît comme l'un des aspects les plus fréquemment critiqués dans les rapports d'audit de l'AUQA.

Les universités tentent de répondre à ce problème de deux manières. Premièrement, la plupart disposent de procédures de doléance, mais, selon l'AUQA, pour plusieurs d'entre elles, elles doivent être révisées (soit en substance, soit en terme de portée auprès de la communauté universitaire). Deuxièmement, une majorité d'universités est en train de s'engager dans une stratégie active de tenue d'un registre officiel des directeurs de thèse (formal Registers of Supervisors). Ces registres garantissent que seuls les membres de la faculté répondant à certains critères sont habilités à être directeurs de thèse. Dans la pratique, il semble qu'il faille qu'un membre de la faculté ait co-dirigé une thèse jusqu'à sa soutenance et ait participé à des activités professionnelles relevant 
de la direction de thèses, avant de pouvoir accéder à la fonction de directeur à part entière. Par ailleurs, leur niveau de qualification doit être supérieur ou égal à celui de l'étudiant dirigé. Dans les meilleurs systèmes, on trouve aussi l'obligation de participer régulièrement à des activités développant l'aptitude à diriger, et l'instauration d'un contrôle du travail de direction des thèses afin de s'assurer que les étudiants reçoivent une attention suffisante (et que la charge de travail des personnels reste raisonnable). Douze universités (32\%) tiennent un tel registre, bien que tous ne soient pas aussi exhaustifs que ce que nous venons de décrire.

Dans la majorité des universités, il existe des opportunités de formation professionnelle pour les directeurs de thèses. Dans au moins cinq universités, un certain niveau de participation à ces activités est une obligation. La tendance générale montre que les universités sont de plus en plus attentives à ce problème. Lorsque de telles formations ne sont pas proposées, l'AUQA a fermement recommandé que les universités remédient à cette lacune.

Au moins quinze universités (39\%) exigent un minimum de deux directeurs pour chaque étudiant en $H D R$, spécialement au niveau du doctorat (et le nombre de ces universités est peut-être plus élevé car certains rapports d'audit sont ambigus sur ce sujet). Cinq universités (13\%) acceptent qu'il n'y ait qu'un seul directeur, mais encouragent le fait qu'il y en ait deux. La tendance est à deux directeurs, et parfois, ce sont même des panels qui supervisent les recherches (pratique mentionnée par quatre universités). Cependant, même si la présence de deux directeurs est reconnue comme préférable à un seul, pour un certain nombre d'universités, le rôle du second directeur (ou co-directeur) n'est pas défini suffisamment précisément. Il est intéressant de voir que la satisfaction des étudiants en $H D R$ ne semble pas être corrélée au nombre de directeurs, si ce n'est du point de vue de la disponibilité de ces derniers.

Lorsque l'on considère les problèmes spécifiques de financement des bourses et des petites subventions, on voit que même s'ils varient d'une université à l'autre et au sein même d'une université, la tendance est tout de même visiblement, pour les étudiants en $H D R$, aux recherches dans les domaines de concentration de la recherche, autrement dit ceux qui font l'objet d'un financement préférentiel. Par exemple, une université a mis en place «un dispositif de bourses de second cycle qui permet de verser rapidement une bourse aux étudiants dont les recherches portent sur l'un des douze domaines clés. La rapidité du dispositif permet à l'université d'attirer des candidats de grande qualité, et de les garder, ce qui est cohérent avec son orientation stratégique ${ }^{17}$.

Un questionnaire portant sur l'expérience de la recherche dans le second cycle (Postgraduate Research Experience Questionnaire : PREQ ${ }^{18}$ ) vient

17. Rapport d'audit de l'AUQA pour l'Université de Wollongong, 2006, p. 33.

18. Le questionnaire est géré par le conseil des carrières du second cycle d'Australie (Graduate Careers Council of Australia). Voir http://www.graduatecarers.com.au/content/full/view/870/ (dernier accès au site le 3 juillet 2007). 
d'être instauré à l'échelle nationale, afin de récolter des données étayées sur les points de vue des étudiants de second cycle. Pour la plupart des universités, le taux de réponse était trop bas pour être exploitable d'un point de vue statistique, et le $P R E Q$ doit encore gagner la confiance du milieu universitaire. Malgré tout, toutes les universités y participent et prennent certains résultats comme point de référence. Indépendamment des résultats du questionnaire, cela témoigne de la volonté des universités de prendre en compte l'opinion de leurs étudiants. Par ailleurs, au moins neuf universités (24\%) ajoutent à ces données d'autres données obtenues par le biais de leurs propres études internes concernant le niveau de satisfaction des étudiants en $H D R$ tant qu'ils suivent encore des cours.

Les étudiants sont de mieux en mieux épaulés avec des services de soutien pour leur admission en $H D R$ et des services de soutien académique. Dans certaines universités, ces pratiques doivent être renforcées (et en particulier rendues plus accessibles), mais en général, les étudiants disposent de toute une gamme de ressources, comme le «livret de ressources pour se préparer à l'admission et ses attentes» (Université de Queensland), les «projets d'apprentissage de la recherche» (Université de Canberra), ou les «sept secrets pour réussir brillamment son doctorat» (Université Flinders).

Les thèses de doctorat sont en principe notées par un panel comprenant au moins deux universitaires éminents extérieurs à l'université, et de préférence venant de l'étranger. Les candidats ne savent pas qui sont les examinateurs avant de connaître leurs résultats.

Un certain nombre d'universités (dont la RMIT, l'Université de Technologie de Queensland et l'Université de Technologie de Sydney) sont en train d'élaborer les moyens d'opérer un contrôle systématique de la qualité dans tous les domaines concernant le HDR. À ce jour, les résultats sont variables.

\section{Le lien entre la recherche et l'enseignement}

Presque toutes les universités australiennes, et peut-être même toutes, affirment que leur recherche apporte quelque chose à leur enseignement, même si cette affirmation est parfois implicite. Néanmoins, l'un des thèmes qui ressort clairement de l'analyse des rapports d'audit de l'AUQA est que ce «lien », s'il est généralement souhaité, n'est ni bien compris, ni systématiquement favorisé. Bien souvent, la responsabilité incombe à la capacité des individus à créer une relation de symbiose entre l'enseignement et la recherche.

Pris dans leur ensemble, les rapports d'audit témoignent de nombreux excellents exemples de liens entre la recherche et l'enseignement, tels que l'inclusion dans le contenu du cours de recherches menées par le personnel chercheur (les étudiants ayant ainsi la chance de pouvoir s'entretenir avec des personnes qui travaillent à la pointe de la recherche dans un domaine donné), 
l'existence de cours de méthodologie de la recherche, et de projets de recherche collectifs. Cependant, même lorsque l'une des grandes universités fait une déclaration formelle de mesures à prendre dans ce domaine ou en définit les grandes lignes, il demeure « un manque de compréhension collectif de ce que signifie un enseignement sous-tendu par la recherche» (rapport d'audit de l'AUQA pour l'Université de Melbourne, 2006, p. 24). Il semblerait donc qu'il soit bien difficile d'ancrer une culture du lien entre la recherche et l'enseignement dans un établissement. De nombreuses universités ont fait part de leur intention de poursuivre leurs efforts dans ce domaine, et c'est en effet l'une des recommandations les plus fréquentes de l'AUQA.

La production de recherche de haut niveau a été prolifique dans le secteur universitaire australien. La plus grande partie a été générée par les universités du Go8, mais il émerge clairement un potentiel de recherche de grande qualité, puisque trente et une autres universités prennent leur essor et mettent l'accent sur le contrôle de qualité.

Les défis sont les mêmes pour toutes et sont le reflet de défis internationaux identiques. L'existence d'un système d'audit de qualité national s'avère précieux, non seulement parce qu'il focalise une plus grande attention sur ces défis, mais aussi pour les succès que les universités remportent en s'efforçant de les relever.

Les auteurs peuvent seulement spéculer sur l'impact que l'introduction du Cadre de qualité de la recherche $(R Q F)$ aura sur la performance des activités de recherche et la manière dont elles sont gérées et menées dans le secteur universitaire. D'autres analyses similaires pourraient être effectuées par la suite pour se rendre compte des changements survenus en matière de qualité et d'impact, et pour confirmer s'ils vont en effet dans la direction souhaitée par ceux qui ont élaboré le Cadre de qualité de la recherche.

\section{BibLIOGRAPHIE}

CLARK B.R. (1998): Creating entrepreneurial universities: Organisational pathways of transformation. New York: Pergamon.

Deakin University Media Statement (6th June 2006) : Statement regarding "teaching intensive" positions at Deakin University http://www.deakin.edu.au/news/upload/ 060607teachingintensive.pdf/, consulté le 2 juillet 2007.

DEEM R. (1998): "New managerialism" and higher education: The management of performance and culture in universities in the United Kingdom. International Studies in the Sociology of Education, 8 (1), 47-69.

GIBBS P. (2001): Higher education as a market: A problem or solution? Studies in Higher Education, 26 (1), 85-94. 
OECD (2001): Changing higher education policies for Japanese National Universities, Higher Education Management, 12 (3), 25-34.

JONGBLOED Ben \& VOSSENSTEYN Hans (2001): "Keeping up Performances: an international survey of performance-based funding in higher education". Journal of Higher Education Policy and Management, 23 (2), 127-145.

RQFDAG: Development Advisory Group for the RQF (October 2006): Research Quality Framework: Assessing Quality and Impact, The Recommended RQF, Commonwealth of Australia.

SHARMA R. (1995) : Program and Graduate Cost Model: An Australian Case Study. JIRA, 4 (1).

SHARMA R. (2004) : Performance-Based Funding in the Entrepreneurial North American and Australian Universities. Journal of Higher Education Policy and Management, 26 (1), 109-118.

WATT C., LANCASTER C., GILBERT J. and HIGERD T. (2004) : Performance funding and quality enhancement at three research universities in the United States. Tertiary Education and Management, 10 (1), 61-72. 
